\title{
Room-temperature metal stamping by microfluidics
}

\author{
S. Sabella ${ }^{\mathrm{a}, 1}$, S. Shiv Shankar ${ }^{\mathrm{a}, 1}$, G. Vecchio $^{\mathrm{b}}$, V. Brunetti $^{\mathrm{b}}$, L. Rizzello ${ }^{\mathrm{b}}$, A. Qualtieri ${ }^{\mathrm{b}}$, L. Martiradonna ${ }^{\mathrm{a}}$, \\ R. Cingolani ${ }^{\text {a }}$, P.P. Pompa ${ }^{\text {a,* }}$ \\ a Italian Institute of Technology, Center for Bio-Molecular Nanotechnology, Via Barsanti, 1, 73010 Arnesano, Lecce, Italy \\ ${ }^{\mathrm{b}}$ National Nanotechnology Laboratory of CNR-INFM, University of Salento, Scuola Superiore ISUFI, Via Arnesano, 16, 73100 Lecce, Italy
}

\section{A R T I C L E I N F O}

\section{Article history:}

Received 22 September 2009

Accepted 30 September 2009

Available online 8 October 2009

\section{Keywords:}

Patterning

Metals

Microscale

Microfluidics

\begin{abstract}
A B S T R A C T
We show the possibility to fabricate highly controlled metal micropatterns on a variety of substrates, such as semiconducting or metallic materials, exploiting a combination of spontaneous galvanic displacement reactions with microfluidics. The process is reliable and quite versatile and allows the fabrication of complex patterns of different metals on a number of substrates in few minutes on a conventional laboratory bench.
\end{abstract}

(c) 2009 Elsevier B.V. All rights reserved.
Micro- and nanofabrication of metal patterned substrates is of high relevance for electronic industry as well as for recent developments in photonics, tissue engineering and material science [1-7]. Several efficient methods, such as electron beam, ion beam, X-ray and photolithography [8] pulsed laser lithography [9] and scanning probe lithography [10-12], enable controlled micro- and nanofabrication of patterned substrates. However, though these techniques can potentially deliver high-resolution features with good reliability, they are expensive and time consuming, typically requiring complex equipment and specialized personnel, with relatively low throughput. Metal patterning has also been demonstrated using self-assembly, microcontact printing [13-15], micromolding in capillary (MIMIC) [16,17], reaction diffusion etching [18], dewetting [19], and other innovative approaches [20-22], although these techniques often rely on a series of fabrication steps that are highly time consuming and cumbersome. In this frame, there is, hence, the necessity to develop alternative strategies that enable easy bench-top fabrication of metal micropatterns with high reproducibility and quick processes. In this work, we demonstrate the possibility to fabricate highly controlled metal patterns on a variety of substrates, such as silicon wafers or other metal-coated planar substrates, by combining spontaneous galvanic displacement reactions (SGDR) with MIMIC. We show that microchanneled plastic stamps can reliably guide the precise localization of the metallization processes on the substrates. Also, the plastic stamps can be used repeatedly, thus obtaining a rapid stamping of the same metallic pattern on several substrates or even

\footnotetext{
* Corresponding author. Tel.: +39 0832 295714; fax: +39 0832295708. E-mail address: piero.pompa@unile.it (P.P. Pompa).

${ }^{1}$ Equally contributing authors.
}

superimposed patterns of one or different metals onto a single substrate easily and in few minutes.

The basic principle underlying the proposed technique is to spatially confine the metallization process with microscale resolution, by limiting the contact area of a reactant solution with the substrate. To achieve this, we positioned patterned PDMS stamps onto the substrates to define a net of microchannels, to be filled with a suitable reactant solution. By this procedure, metallization occurs via SGDR, only within the boundaries of the microchannels. Besides the ease of execution (e.g., no surface modifications are required), the process ensures that the unmetalized part of the substrate remains pristine, avoiding the presence of any residual resist material, unlike other lithographic techniques. Compared to other approaches aimed at the fabrication of metalized patterns through SGDR [23], our method has broader applicability, since it can be applied to fast reacting systems and, in particular, to the realization of superimposed metal patterns on a single substrate. Fig. 1 shows a scheme of the process based on suitable plastic replicas that leads to precise metal stamping onto a wide variety of substrates. First, the stamp is lightly pressed onto the substrate (Fig. 1a), then it is filled by an appropriate metal ion solution (Fig. 1b). As a consequence, metallization occurs only in the regions of the substrate that are exposed to the reaction (Fig. 1c). The only requirement here is that the reduction potential of the metal ion solution injected into the microchannels is sufficiently high to react with the substrate and get deposited in the metallic form [2]. Once the reaction of the metal ions with the substrate is over (typically after few minutes), the microchannels are injected with deionized water to quench the reaction and to wash the substrate. Finally, after suction of the water from the channels, the PDMS stamp is carefully removed, the substrate is washed again with deionized water and dried under flowing nitrogen. A wide variety of substrates can be metalized by this 

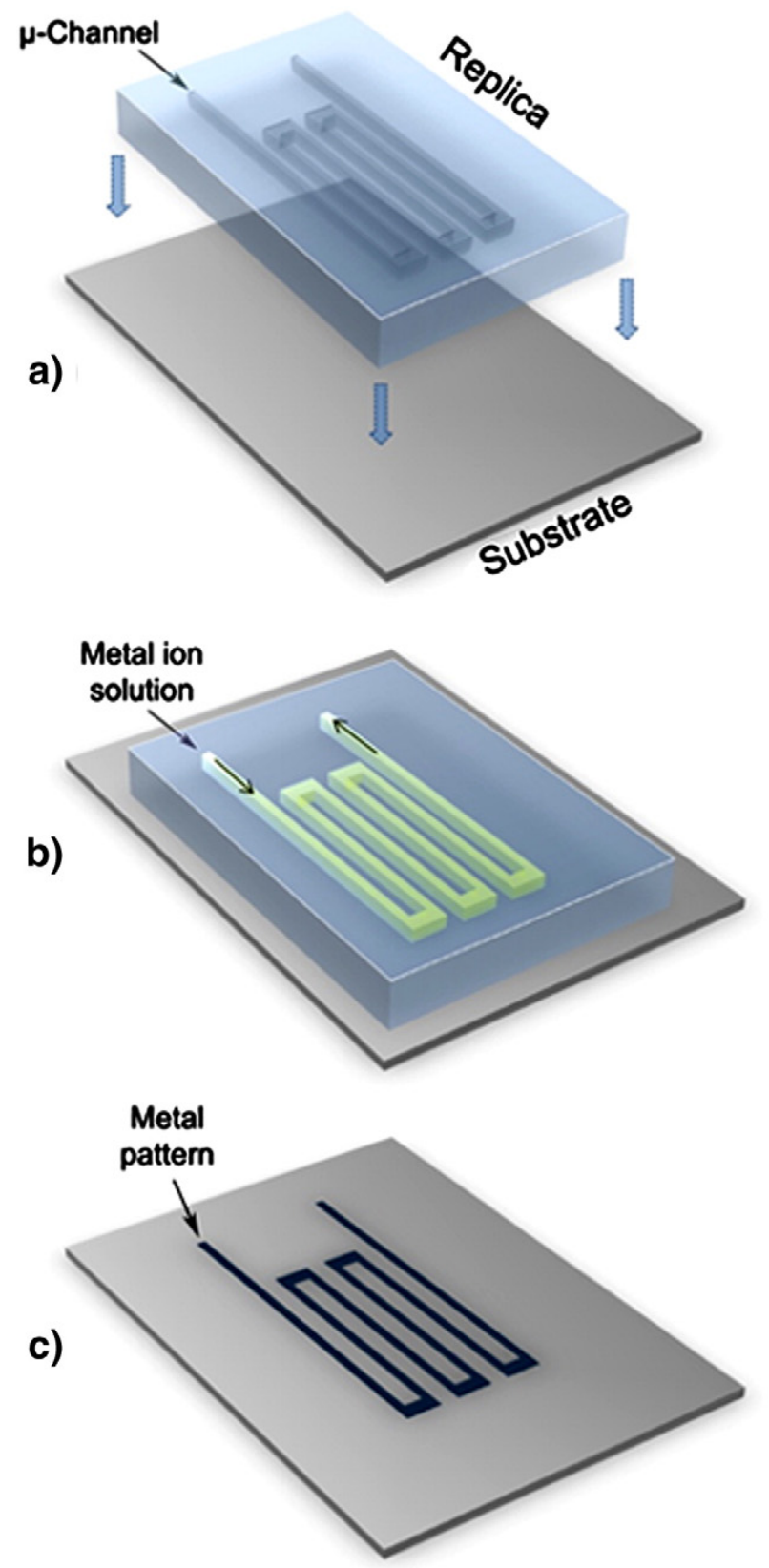

Fig. 1. Schematic showing the process of fabrication of a metalized pattern on a substrate using a combination of replica mold and galvanic reaction. a) A replica mold with open continuous channels is first pressed against the substrate; b) an appropriate solution of metal ions capable of galvanically reacting and getting deposited on the substrate is injected through the microfluidic channel and left to react for few minutes: c) after few minutes the metal ion solution is washed out and the replica gently removed to obtain the pattern.

approach with arbitrary patterns. The degree of metallization can be directly controlled by controlling the amount of metal ions in the solution and the time of reaction (typical processes usually take no longer than $2 \mathrm{~min}$ ).

As a first example, we demonstrated the fabrication of gold and silver patterns on a p-type silicon substrate. After placing the PDMS stamp onto the silicon substrate, a solution of $\mathrm{HAuCl}_{4}$ in $\mathrm{HF}$ and $\mathrm{NH}_{4} \mathrm{~F}$ mixture [2] is passed through the channels, and allowed to react for 1$2 \mathrm{~min}$. Within this short reaction time, a thin layer of metallic gold is uniformly and selectively deposited in the regions delimited by the microchannels. Fig. 2a and b shows representative optical images of different patterns of gold on silicon wafer. The gold stripe patterns are
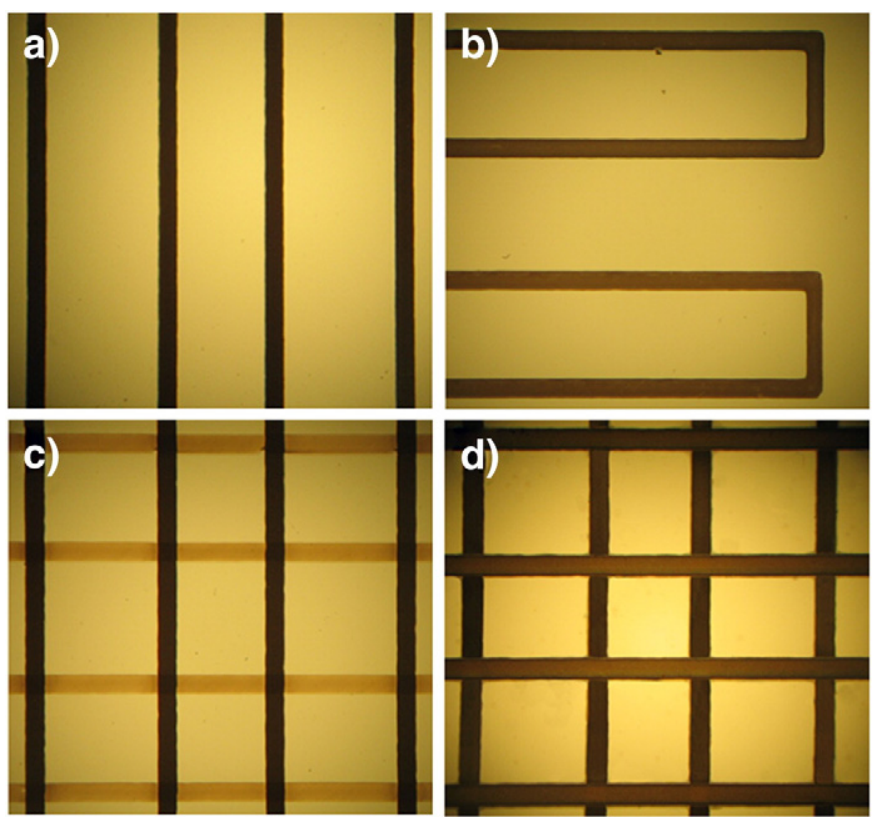

Fig. 2. Optical micrographs of a) and b) two representative gold patterns on a silicon substrate; c) bimetallic gold/silver grid pattern on a silicon substrate; d) gold grid pattern on a $50 \mathrm{~nm}$ thick silver film coated onto a glass slide. All the stripes in (a-d) were $80 \mu \mathrm{m}$ wide.

$80 \mu \mathrm{m}$ wide. It can be noticed that the metalized patterns are spatially well defined without any sign of diffusion under the edges of the plastic microchannels. Once a particular metalized pattern is realized on a substrate, the process can be repeated on the same substrate, thus superimposing any other pattern of the same or different metal. This is demonstrated in Fig. 2c where we show crossed gold and silver patterns on silicon. In this case, a stripe pattern of silver was first fabricated on a silicon substrate by using a reactant solution of $\mathrm{AgNO}_{3}$ in $\mathrm{HF}$ and $\mathrm{NH}_{4} \mathrm{~F}$ [2]; after washing and drying, a second similar pattern of gold was realized by placing the PDMS stamp perpendicular to the previous silver pattern and following the usual reaction conditions. Importantly, no intermediate lithographic steps or complex procedures are required to fabricate such two-metal patterns with microscale resolution. Moreover, our strategy relies on a direct reaction of the metal ions with the surface of the substrate, followed by local deposition within the microchannels. This ensures a very good contact of the overlying metal pattern with the underlying silicon, as the metal is deposited by etching out the surface oxide layer and directly displacing the elemental silicon beneath it.

Apart from the fabrication of patterns on silicon substrates, metal patterns can also be realized directly onto metal surfaces. Fig. $2 \mathrm{~d}$ shows a representative image of a pattern of gold over a silver thin film. In this case, a $50 \mathrm{~nm}$ thick film of silver was first thermally vacuum deposited over a glass (or silica) substrate and then covered with the PDMS stamp. The microchannels were subsequently filled with aqueous $\mathrm{HAuCl}_{4}$ solution and allowed to react for 2 min before washing with water. The grid pattern in Fig. 2d was obtained by repeating the patterning process with the stamp put perpendicular to the first pattern and following the reaction with the same $\mathrm{HAuCl}_{4}$ aqueous solution. Such bimetallic pattern is similar to those recently achieved by our group for different applications, although this latter was fabricated using a more complex procedure based on photoresists and electron beam or optical lithographies [24,25].

To better assess the morphology of the fabricated patterns, we performed scanning electron microscope (SEM) analyses. Fig. 3a and b shows representative SEM images of a large gold pattern on a silicon wafer, showing the features at one end of the pattern. It can be observed that even the corners of the microchannel in the stamp are 

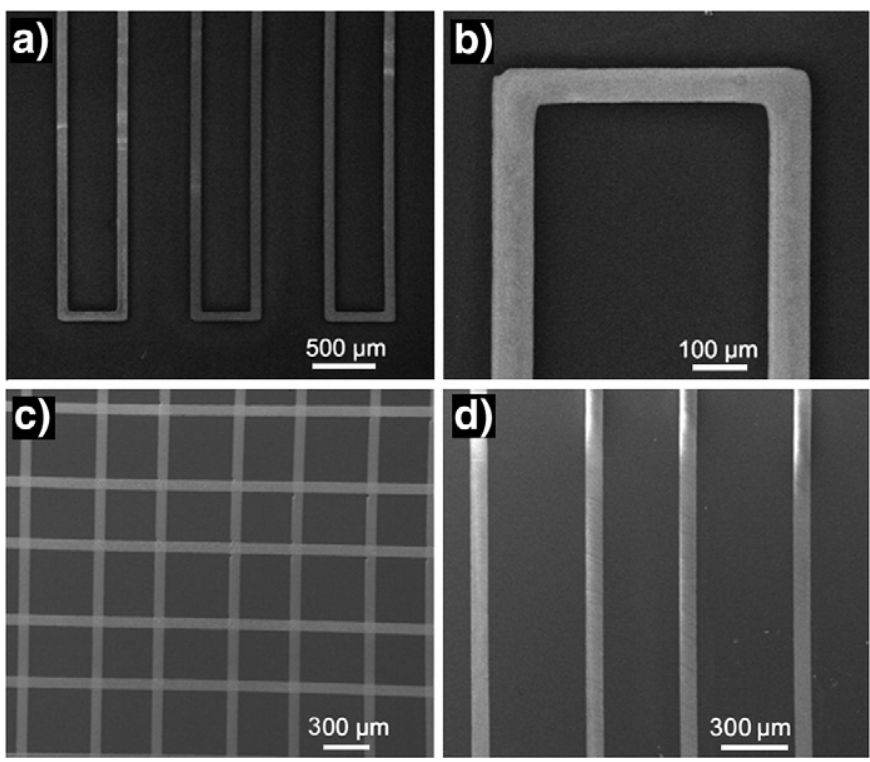

Fig. 3. Scanning electron microscope (SEM) images of various metal pattern substrates. $a, b)$ Representative SEM images of a gold pattern on silicon substrate at different magnifications. c) SEM image of a grid pattern consisting of perpendicular gold (vertical) and silver (horizontal) stripes on silicon. d) SEM image of gold stripe pattern on a nickel coated silica substrate. All the stripes in (a-d) were $80 \mu \mathrm{m}$ wide.

precisely reproduced on the substrate. Fig. $3 c$ is a SEM image of the $\mathrm{Au}-\mathrm{Ag}$ grid pattern on a silicon substrate, corresponding to the sample shown in the optical image of Fig. 2c. The metallic stripes show a not perfectly flat and smooth surface. It is known, in fact, that in SGDR the deposition of the metal on the silicon substrate in the presence of $\mathrm{HF}$ is governed by the following reaction [2]:

$4 M^{n+}(\mathrm{aq})+n S i^{0}(\mathrm{~s})+6 n F^{-}(\mathrm{aq})=4 M^{0}(\mathrm{~s})+n \operatorname{SiF}_{6}^{2-}(\mathrm{aq})$

From the above equation, it can be readily inferred that the amount and rate of metal deposited are dependent on the oxidation state of its corresponding metal ion solution used for the galvanic displacement reaction. This can lead to minor differences in the surface structure within the two-metal stripe regions. However, by properly tailoring the SGDR reaction parameters (concentration of the species, incubation time, etc.), it is possible, if required, to precisely control the surface morphology of the different metal regions, obtaining, for instance, flat or uniform and controlled nanostructured surfaces $[24,25]$

After getting encouraging results from gold and silver patterns on silicon substrates, and of one noble metal over another noble metal surface, we also demonstrated the possibility to fabricate metal patterns that could act as potential candidates for patterned magnetic materials. To this purpose, we used a silica substrate, covered by a $50 \mathrm{~nm}$ thick layer of nickel, and $\mathrm{HAuCl}_{4}$ as the reactant solution. The rest of the process was similar to that described above. Galvanic displacement of nickel substrate by copper has been earlier demonstrated, emphasizing their catalytic properties [26]. However, patterned deposition over such substrates has not yet been demonstrated, thus paving the way for new potential applications. In Fig. 3d, a SEM image of such a substrate with a gold stripes pattern on a nickel coated substrate can be observed. Clearly, the stripes are well defined as observed in the previous cases. This example proves that the technique described here is equally efficient for a wide variety of systems and, thus, is quite versatile.

Finally, we investigated in more detail the spatial control of the reaction by an elemental mapping of the different species on the patterned substrates, using energy dispersive X-ray microanalysis
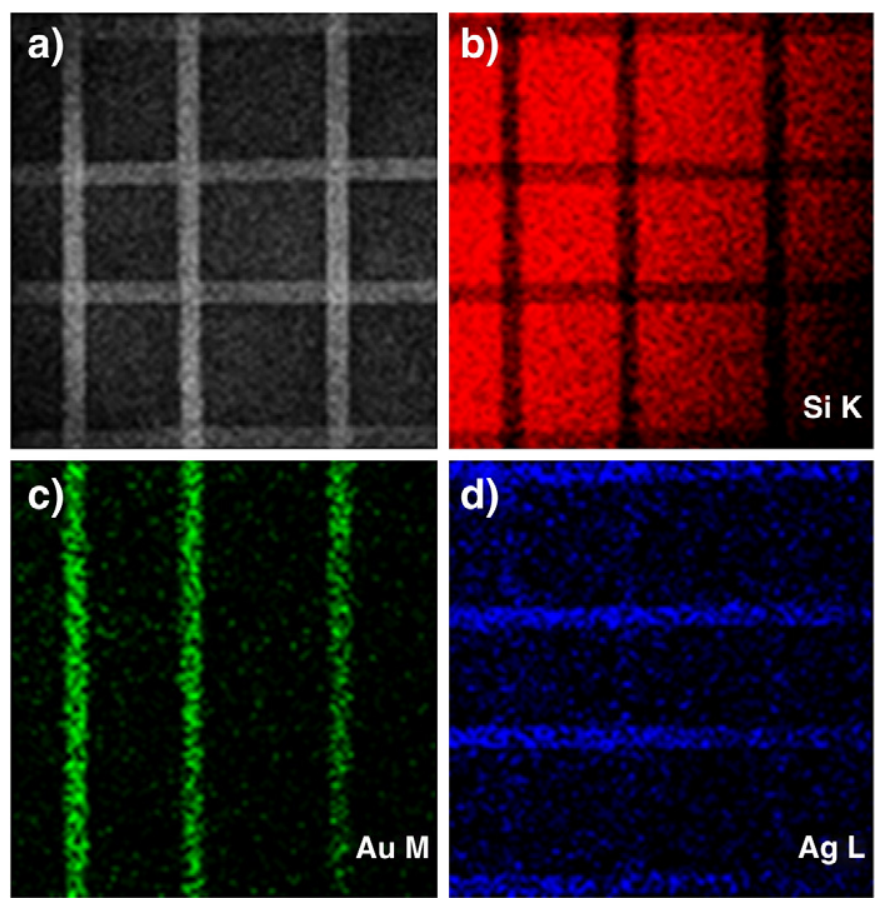

Fig. 4. EDAX elemental distributions analysis of the gold-silver patterned silicon substrate. a) SEM image of the area used for the elemental analysis. b) Silicon distribution on the surface of the substrate, imaged from the $\mathrm{K}$ line of the silicon species. c) Gold distribution on the surface imaged from the M line of the gold species. d) Silver distribution imaged from the $\mathrm{L}$ line of the silver species.

(EDAX). The area of the Au/Ag grid pattern, studied for mapping the elemental distribution on the patterned substrate, is shown in Fig. 4a (SEM image). Fig. 4b-d reports, in false colors, the spatial distributions of $\mathrm{Si}, \mathrm{Au}$ and $\mathrm{Ag}$, respectively, on the sample surface. In Fig. 4b, it can be noticed that a strong reduction of the $\mathrm{Si} \mathrm{K}$ line signal is obtained in correspondence of the patterned region. This indicates that, after the Galvanic displacement reaction ends, the substrate under the microchannel region is covered by a thick layer of metal concealing well the underlying silicon part. Similarly, from Fig. $4 \mathrm{c}$ and d, it can be confirmed that the two metals, gold and silver, have been selectively deposited in the predetermined regions only. In particular, gold stripes preserve a uniform width also in the presence of raised regions, as in the case of crossings with $\mathrm{Ag}$ stripes. This proves that the sealing effect of PDMS on the substrate is verified also on rough or terraced substrates, giving further generalizability to our approach.

In conclusion, we have demonstrated that the use of capillary microchanneled PDMS stamps is a feasible way to readily fabricate micropatterns of single or multiple metals on a single galvanically reactive substrate. The process is quite versatile and can be applied on a wide range of semiconducting, metallic or even magnetic substrates without resorting to high end instruments. Each fabrication process can be realized in few minutes time conveniently on a conventional laboratory bench, and can be reliably replicated on a number of substrates using a single polymeric stamp of desired microchanneled patterns. As a way ahead, we would like to use this technique to fabricate patterned substrates for tissue engineering, cell patterning and for the patterned assembly of magnetic materials (or magnetic NPs-labeled biomolecules) under the influence of magnetic field, exploiting the underlying magnetic metal pattern.

\section{Appendix A. Supplementary data}

Supplementary data associated with this article can be found, in the online version, at doi:10.1016/j.matlet.2009.09.066. 


\section{References}

[1] Andrew P, Barnes WL. Molecular fluorescence above metallic gratings. Phys Rev B 2001;64:125405.

[2] Carraro C, Maboudian R, Magagnin L. Metallization and nanostructuring of semiconductor surfaces by galvanic displacement processes. Surf Sci Rep 2007:62:499-525.

[3] Xia Y, Whitesides GM. Soft lithography. Angew Chem Int Ed 1998;37:550-75

[4] Haynes CL, Van Duyne RP. Nanosphere lithography: a versatile nanofabrication tool for studies of size-dependent nanoparticle optics. J Phys Chem B 2001;105:5599-611.

[5] McAlpine MC, Friedman RS, Lieber CM. Nanoimprint lithography for hybrid plastic electronics. Nano Lett 2003;3:443-5.

[6] Xu Q, Bao J, Rioux RM, Perez-Castillejos R, Capasso F, Whitesides GM. Fabrication of large-area patterned nanostructures for optical applications by nanoskiving. Nano Lett 2007;7:2800-5.

[7] Khang D, Lu J, Yao C, Haberstroh KM, Webster TJ. The role of nanometer and submicron surface features on vascular and bone cell adhesion on titanium. Biomaterials 2008;29:970-83.

[8] Wallraff GM, Hinsberg WD. Lithographic imaging techniques for the formation of nanoscopic features. Chem Rev 1999;99:1801-22.

[9] Kerner G, Asscher M. Buffer layer assisted laser patterning of metals on surfaces. Nano Lett 2004;4:1433-7.

[10] Soh HT, Guarini KW, Quate CF. Scanning probe lithography. Boston: Kluwer Academic Publishers; 2001. p. 37-130.

[11] Marrian CRK, Perkins FK, Brandow SL, Koloski TS, Dobisz EA, Calvert JM. Low voltage electron beam lithography in self-assembled ultrathin films with the scanning tunneling microscope. Appl Phys Lett 1994;64:390-2.

[12] Basnar B, Willner I. Dip-pen-nanolithographic patterning of metallic, semiconductor, and metal oxide nanostructures on surfaces. Small 2009;5:28-44.

[13] Xia Y, Rogers JA, Paul KE, Whitesides GM. Unconventional methods for fabricating and patterning nanostructures. Chem Rev 1999;99:1823-48.

[14] Burdinski D, Blees MH. Thiosulfate- and thiosulfonate-based etchants for the patterning of gold using microcontact printing. Chem Mater 2007;19:3933-44.
[15] Loo YL, Willett RL, Baldwin KW, Rogers JA. Interfacial chemistries for nanoscale transfer printing. J Am Chem Soc 2002;124:7654-5.

[16] Kim E, Xia Y, Whitesides GM. Polymer microstructures formed by moulding in capillaries. Nature 1995;376:581-4.

[17] Kim E, Xia Y, Whitesides GM. Micromolding in capillaries: applications in materials science. J Am Chem Soc 1996;118:5722-31.

[18] Grzybowski BA, Bishop KJM. Micro- and nanoprinting into solids using reactiondiffusion etching and hydrogel stamps. Small 2009;5:22-7.

[19] Radha B, Kulkarni GU. Dewetting assisted patterning of polystyrene by soft lithography to create nanotrenches for nanomaterial deposition. ACS Appl Mater Interf 2009;1:257-60.

[20] Sun Z, Li Y, Wang Y, Chen X, Zhang J, Zhang K, et al. Three-dimensional colloidal crystal-assisted lithography for two-dimensional patterned arrays. Langmuir 2007;23:10725-31.

[21] Chou SY, Keimel C, Gu J. Ultrafast and direct imprint of nanostructures in silicon. Nature 2002;417:835-7.

[22] Black AJ, Paul KE, Aizenberg J, Whitesides GM. Patterning disorder in monolayer resists for the fabrication of sub-100-nm structures in silver, gold, silicon, and aluminum. J Am Chem Soc 1999;121:8356-65.

[23] Porter LA, Choi HC, Schmeltzer JM, Ribbe AE, Elliott LCC, Buriak JM. Electroless nanoparticle film deposition compatible with photolithography, microcontact printing, and dip-pen nanolithography patterning technologies. Nano Lett 2002;2:1369-72.

[24] Shankar SS, Rizzello L, Cingolani R, Rinaldi R, Pompa PP. Micro/nanoscale patterning of nanostructured metal substrates for plasmonic applications. ACS Nano 2009;3:893-900.

[25] Rizzello L, Shankar SS, Fragouli D, Athanassiou A, Cingolani R, Pompa PP. Microscale patterning of hydrophobic/hydrophilic surfaces by spatially controlled galvanic displacement reactions. Langmuir 2009;25:6019-23.

[26] Bansal V, Jani H, Plessis JD, Coloe PJ. Bhargava SK Galvanic replacement reaction on metal films: a one-step approach to create nanoporous surfaces for catalysis. Adv Mater 2008:20:717-23. 